\title{
FIELD EVALUATION OF SPATIAL REPELLENCY OF METOFLUTHRIN- IMPREGNATED PLASTIC STRIPS AGAINST ANOPHELES GAMBIAE COMPLEX IN BAGAMOYO, COASTAL TANZANIA
}

\author{
HITOSHI KAWADA, ${ }^{1}$ EMMANUEL A. TEMU, ${ }^{1}$ JAPHET N. MINJAS, ${ }^{2}$ OSAMU MATSUMOTO ${ }^{3}$ \\ TOMONORI IWASAKI ${ }^{4}$ AND MASAHIRO TAKAGI ${ }^{1}$
}

\begin{abstract}
Metofluthrin is a newly synthesized pyrethroid possessing high knockdown and lethal activity against mosquitoes. Studies of metofluthrin-impregnated plastic strips have been performed with dengue vectors. This study reports the efficacy of the new prototypes of metofluthrin-impregnated plastic strips against malaria vectors, Anopheles gambiae complex, in the Kongo villages of Bagamoyo district in coastal Tanzania. The study, using 20 houses, half intervention, half control, was conducted for a 124-day period. Pyrethrum spray sheets and Centers for Disease Control and Prevention light traps were used to sample mosquito population indices. The mosquito density indices of the intervention houses were observed to be significantly lower than those of the control houses when pyrethrum spray sheet collection was used ( $F$ $=4.61,1 \mathrm{df}, P=0.038 ; 98.7 \%$ reduction of total mosquito collection compared with that for the controls). These low indices were observed despite the large openings found in Bagamoyo houses, which were predicted to have a considerable negative effect on the spatial repellency of metofluthrin. Based on the present results, the pyrethrum spray sheet collection was the better of the 2 collection methods.
\end{abstract}

KEY WORDS SumiOne ${ }^{\mathrm{TM}}$, pyrethroid, malaria vector, mosquito population sampling, traps

\section{INTRODUCTION}

Metofluthrin (SumiOne $\left.{ }^{\circledR}\right), 2,3,5,6$-tetrafluoro4-methoxymethylbenzyl(E:Z $\approx 1: 8)(1 R, 3 R)-2,2$ dimethyl-3-(prop-1-enyl)cyclopropanecarboxylate, is a newly synthesized pyrethroid produced by Sumitomo Chemical Co., Ltd. (Osaka, Japan). The high knockdown and lethal activity of metofluthrin against mosquitoes has been documented previously (Ujihara et al. 2004, Matsuo et al. 2005). The high vapor pressure of metofluthrin $\left(1.87 \times 10^{-3} \mathrm{~Pa}\right.$ at $\left.25^{\circ} \mathrm{C}\right)$, which is 2-fold and 100 fold greater than those of $d$-allethrin and permethrin, respectively, enables vaporization at ambient temperature, whereas other conventional pyrethroids require heating for evaporation. The unique characteristics of metofluthrin may result in novel mosquito controlling devices and strategies that require no external energy for vaporization, long-term efficacy, and low maintenance costs.

A simple prototype device with metofluthrinimpregnated multilayer paper strips showed promising spatial repellency against mosquitoes in laboratory and field studies (Kawada et al. 2004a, 2004b). Under simulated outdoor condi-

${ }^{1}$ Department of Vector Ecology \& Environment, Institute of Tropical Medicine, Nagasaki University, Nagasaki, Japan.

${ }^{2}$ Muhimbili University College of Health Science, Dar es Salaam, Tanzania.

${ }^{3}$ Agricultural Chemicals Research Laboratory, Sumitomo Chemical Co., Ltd., Hyogo, Japan.

${ }^{4}$ Environmental Health Division, Sumitomo Chemical Co., Ltd., Osaka, Japan. tions, mosquitoes (Anopheles sp. and Culex sp.) were repelled by airborne metofluthrin vapors (Kawada et al. 2004a). The field tests suggested that metofluthrin may be a suitable spatial repellent leading to the reduction of mosquito bites for Anopheles sundaicus (Rodenwaldt), An. balabacensis (Baisas), and Culex quinquefasciatus (Say) in shelters without walls (beruga), such as those used by people in Lombok Island, Indonesia, which are associated with high risk of malaria transmission (Kawada et al. 2004b). To increase the effectiveness of metofluthrin, we manufactured a cylindrical slow-release plastic device consisting of a 20-g strip impregnated with $1,000 \mathrm{mg}$ metofluthrin. Use of this device resulted in spatial repellency for $14 \mathrm{wk}$, with 4 strips used per beruga in Lombok (Kawada et al. 2005a). Further, spatial repellency of this device was achieved indoors against Aedes aegypti (L.) in residences of Do Son, Hai Phong city, Vietnam (Kawada et al. 2005b). However, the effective duration (6 wk at 1 strip per room) appeared insufficient for practical use. We hypothesized that long-term effectiveness of these devices may be achieved by 1) designing devices of different shapes, 2) adopting formulations with different optimal compositions and densities of the plastic polymer in order to reduce the release rate of the active ingredient, 3 ) increasing the concentration of the active ingredient, and 4) increasing the number of strips per room.

Accordingly, we manufactured a new latticework plastic strip that was designed to reduce the release rate of metofluthrin by approximately $50 \%$ compared to the previous device. The new 
latticework strips contained $600 \mathrm{mg}$ metofluthrin per 12.3-g strip, and tested at 1 strip per 2.6$5.5 \mathrm{~m}^{2}$ were effective for at least 8 wk against $A e$. aegypti in the residential houses in My Tho city, Tien Giang province, Vietnam (Kawada et al. 2006).

Numerous field trials in residential houses have assessed the effectiveness of metofluthrin-impregnated plastic strips against dengue vectors; however, we have not performed similar studies on malaria vectors in malaria endemic or epidemic areas. The present study reports the efficacy of the new prototypes of metofluthrinimpregnated plastic strips against the An. gambiae complex in Bagamoyo, coastal Tanzania.

\section{MATERIALS AND METHODS}

Metofluthrin-impregnated latticework plastic strips: New plastic strips were supplied by Sumitomo Chemical Co. Ltd. (Hyogo, Japan). The area of the plastic latticework structure was 9 $\times 18 \mathrm{~cm}^{2}$; thickness, $0.2 \mathrm{~cm}$; and weight, $10.7 \mathrm{~g}$. The strip was made of polyethylene and impregnated with $5 \%(\mathrm{w} / \mathrm{w})$ metofluthrin.

Study location: The study was conducted in the malaria holoendemic Kongo villages of Bagamoyo $\left(06^{\circ} 32^{\prime} 44^{\prime \prime} \mathrm{S}, 38^{\circ} 50^{\prime} 03^{\prime \prime} \mathrm{E}\right.$, center of study area), which is located $70 \mathrm{~km}$ northwest of Dar es Salaam in coastal Tanzania. The villages are southwest of the Bagamoyo town, under the Yombo administrative division, with a population of 21,000. The district experiences a hot tropical coastal climate and high relative humidity with little variation in the annual temperatures. A bimodal pattern of rainfall is observed with longer periods of rainfall between April and May and the shorter ones that occur in the months of October and November. The majority of the people residing in Bagamoyo villages are farmers who cultivate cassava, maize, and cashew nuts or have small coconut plantations or rice irrigation farms at subsistence levels. Most residential houses have traditional mud walls and thatched roofs; very few houses are made of cement bricks.

Plasmodium falciparum is the major malaria parasite that accounts for $>95 \%$ of the malaria cases in this area. Both An. gambiae and An. funestus complexes are the major malaria vectors in Bagamoyo (Davis et al. 1995, Shiff et al. 1995, Temu et al. 1998), and their densities fluctuate depending upon the rainfall patterns (Shiff et al. 1995); consequently, malaria transmission is high and occurs throughout the year.

Volunteers from the study sites were recruited, and participated only after informed consent was obtained. We recommended the use of a bed net (not impregnated with insecticide) for all volunteers. Bed nets were provided in the event that the people in the intervention and control houses did

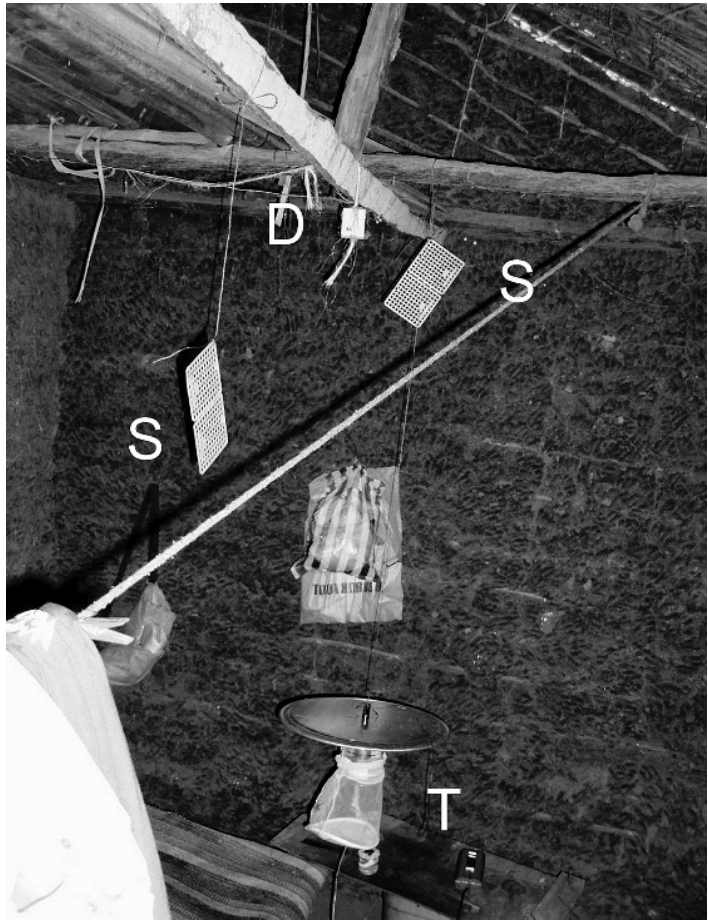

Fig. 1. Treatment of metofluthrin-impregnated plastic strips in a room, Bagamoyo, Tanzania. S, metofluthrin-impregnated plastic strip; D, data logger $\left(\mathrm{HOBO}^{\circledR}\right) ; \mathrm{T}, \mathrm{CDC}$ trap.

not have them; this was done in order to ensure safety of the residents, which was regarded as the top priority. The study protocol was reviewed in accordance with the ethical review process of Muhimbili University College of Health Science, Dar es Salaam, Tanzania and Institute of Tropical Medicine, Nagasaki University, Japan.

Strip treatment and mosquito collection: We randomly selected 20 houses with volunteers that had given informed consent in the trial region. One half of the houses were randomly assigned to the intervention group where strips were used. The other half were randomly assigned as the controls. The selection of houses for intervention and control was performed with the use of the table of random numbers. Intervention with the use of the metofluthrin-impregnated plastic strips was initiated on May 18, 2006. The strips were directly hung from the ceiling, or they were suspended with a rope inside the rooms at 1 strip per $1.0-2.5 \mathrm{~m}^{2}$ (average 1 strip per $1.8 \mathrm{~m}^{2}$, Fig. 1). A total of 115 strips were used for the 10 intervention houses.

Indoor mosquito collection was performed by 2 conventional methods, namely, the pyrethrum spray sheet collection method (Service 1993) and the light-trap collection method with batterypowered Centers for Disease Control and Pre- 
vention (CDC)-type traps (Davis et al. 1995). Pyrethrum spray sheet collection is used commonly as a standard; it is a quick and easy method and is usually regarded as the most efficient of all the available methods for collecting resting mosquitoes (Service 1993). The CDC trap collection was used as a substitute for humanbaited collection. Pyrethrum spray sheet collections were performed in 5 intervention and control houses each; in the remaining 10 houses, the light-trap collection method was employed. Pyrethrum spray sheet collections were performed in the morning $(0600-1000 \mathrm{~h})$ by $3-4$ staff members. Light-trap collections were performed during the night by suspending a single CDC trap with a bulb and no dry ice near the bed, approximately $1-1.5 \mathrm{~m}$ above the floor. Each member of the household participated in the study and was provided adequate instructions regarding the appropriate operation of the light trap, i.e., to switch the trap on at sunset, close the neck of the collection bag at sunrise to prevent the mosquitoes from escaping, and switch the motor off. The traps were collected in the morning and testing was performed to ascertain whether the trap functioned properly throughout the night. Two days before the intervention, a preliminary survey was conducted in order to determine the mosquito density. Light-trap collections were conducted on the day of intervention, and 5, 20, 34, 61, 89, and 124 days after intervention; pyrethrum spray sheet collections were conducted 20,34, 61, 89, and 124 days after the intervention. In the case of pyrethrum spray sheet collection, mosquito collections were not conducted on the day of intervention and 5 days after the intervention. Anopheline mosquitoes were identified and classified morphologically into An. gambiae complex, An. funestus complex, and other species. The number of mosquitoes per house was recorded, and the mosquito density index was calculated.

Measurement of environmental factors: Environmental factors such as room temperature, humidity, floor area, room volume, window area, door area, and areas of other openings (open eaves, etc.) were measured for the 10 metofluthrin-treated houses. In the intervention group, for those houses having several rooms, structural variables such as the floor area, volume, and opening area were calculated as a whole. The corrected open area for each house was calculated as the sum of the window area, half of the door area (since doors were normally closed at night), open eaves area, and area of any other opening. Throughout the trial, the room temperature and humidity were recorded on an hourly basis by using a data logger $\left(\mathrm{HOBO}^{\circledR}\right.$, Onset Computer Corp., Pocasset, MA).

Data analysis: The mosquito density index was calculated as the mean number of female mosquitoes collected per house on a daily basis. Prior to statistical analysis, the number of mosquitoes was $\log _{10}(n+1)$-transformed for normalization. The differences between the number of mosquitoes per house per day in the intervention and the control houses were compared by using a 2-way repeated-measure ANOVA (JMP 7 Japanese Edition, JMP Japan).

\section{RESULTS}

Residual spatial repellency of metofluthrin-impregnated plastic latticework strips against mosquitoes: The dominant species collected at the trial sites were those belonging to the An. gambiae complex, followed by Cx. quinquefasciatus. Additionally, a small number of An. funestus complex, Mansonia sp., and other anopheline mosquitoes were collected. Prior to the intervention, the maximum number of An. gambiae complex mosquitoes collected by using the CDC traps in a house was 963 per night Since the commencement of the study, the proportion of blood-fed mosquitoes was extremely low because most families used a bed net (not impregnated with insecticides).

Table 1 shows the differences between the numbers of female An. gambiae complex mosquitoes collected by using the CDC traps and those collected by using the pyrethrum spray sheet in the metofluthrin-intervention and the control houses. After the intervention with metofluthrin-impregnated strips (5-124 days), the mosquito density indices of the intervention houses were significantly lower than those of the control houses when the collection was performed by using the pyrethrum spray sheet $(F=4.61,1 \mathrm{df}$, $P=0.038 ; 98.7 \%$ reduction in the total mosquito collection when compared with that for the controls). The difference in the mosquito density indices of the An. gambiae complex between the intervention and the control houses was, on the other hand, not significant when the CDC trap collection method ( $F=2.70,1 \mathrm{df}, P=0.11)$ was used. Collection by using both the CDC traps and the pyrethrum spray sheets revealed that the density index of An. funestus complex (0.2-5.4 with CDC trap collection and $0-2.0$ with pyrethrum spray collection, respectively, in the control houses) was lower than that of $A n$. gambiae complex, thereby rendering statistical comparison between the intervention and the control groups nonfeasible.

Environmental factors in the intervention houses where Metofluthrin-impregnated plastic strips were used: The average temperatures and humidities in the intervention houses (from June 20 to August 3, 2006) were $23.9-25.8^{\circ} \mathrm{C}$ and 68.4 $80.0 \% \mathrm{RH}$, respectively. Although the total floor area $\left(6-43.8 \mathrm{~m}^{2}\right)$ and the volume $\left(12-131.3 \mathrm{~m}^{3}\right)$ varied, the factors calculated, including the 
Table 1. Changes in the mosquito density index ${ }^{1}$ by pyrethrum spray sheet catch collection and Centers for Disease Control trap collection in the metofluthrin-intervention and control houses in Bagamoyo, Tanzania.

\begin{tabular}{lcc}
\hline \hline & \multicolumn{2}{c}{ Mosquito density index (Anopheles gambiae s.1.) } \\
\cline { 2 - 3 } Days after intervention & Intervention $(95 \%$ confidence interval) & Control (95\% confidence interval) \\
\hline Pyrethrum spray sheet catch collection & & $2.0(1.0)$ \\
20 & $0.2(0.4)$ & $11.4(5.6)$ \\
34 & $0.2(0.4)$ & $8.0(4.2)$ \\
61 & $0.0(-)$ & $7.2(4.4)$ \\
89 & $0.0(-)$ & $2.4(2.3)$ \\
124 & $0.0(-)$ & $90.0(69.5)$ \\
CDC trap collection & & $136.0(282.1)$ \\
-2 & $246.3(302.9)$ & $34.1(36.5)$ \\
0 & $113.9(74.8)$ & $36.2(30.8)$ \\
5 & $55.6(39.0)$ & $34.6(19.7)$ \\
20 & $24.3(16.8)$ & $26.2(14.1)$ \\
61 & $11.8(11.7)$ & $16.0(10.6)$ \\
89 & $8.4(8.2)$ & $6.2(5.5)$ \\
\hline
\end{tabular}

${ }^{1}$ Mosquito density index $=$ no. of female mosquitoes/house/day.

corrected opening area/volume that indicates the "degree of openness," were distributed over a narrow range from 0.06 to 0.15 . The correlation between the spatial repellent efficacy (expressed as the cumulative number of mosquitoes in each intervention house collected by the pyrethrum spray sheet collection) and the environmental factors was not significant.

Table 2 lists the environmental factors and the effective duration of the metofluthrin-impregnated plastic strips in the present study as well as those of the intervention houses measured in My Tho city, Tien Giang, Vietnam, where a similar metofluthrin trial was conducted in the same season in the year 2005 (Kawada et al. 2006). Variables including the average temperature and humidity were calculated on an hourly basis from June 20 to August 3, 2006 for Bagamoyo and from June 20 to September 4, 2005 for My Tho. The room temperature was lower and the humidity was higher in Bagamoyo houses compared to the corresponding conditions in the My Tho houses. Although the floor areas and the volumes were larger in the houses in My Tho compared to those in Bagamoyo, the corrected opening area per total average volume of the houses in Bagamoyo was almost twice that of houses in My Tho, thereby indicating that the Bagamoyo houses are more "open" than the My Tho houses.

\section{DISCUSSION}

Metofluthrin-impregnated strips significantly reduced the density index of $A n$. gambiae complex in the intervention houses when compared with that observed in the control houses. Generally, the density of An. funestus complex in Bagamoyo area is much lower than that of An. gambiae

Table 2. Environmental factors of the intervention houses and effective duration of metofluthrin-impregnated plastic strips.

\begin{tabular}{|c|c|c|}
\hline \multirow[b]{2}{*}{ Environmental factors } & \multicolumn{2}{|c|}{ Trial sites (year) and target mosquito } \\
\hline & $\begin{array}{c}\text { My Tho (2005), } \\
\text { Aedes aegypti }\end{array}$ & $\begin{array}{l}\text { Bagamoyo (2006), }{ }^{1} \\
\text { Anopheles gambiae s.l. }\end{array}$ \\
\hline Average temperature $\left({ }^{\circ} \mathrm{C}\right)^{3}$ & $29.1(0.8)$ & $24.8(0.7)$ \\
\hline Average humidity $(\% \mathrm{RH})^{3}$ & $70.1(5.1)$ & $75.3(3.9)$ \\
\hline Total floor area $\left(\mathrm{m}^{2}\right) /$ house & $32.1(10.5)$ & $22.0(14.1)$ \\
\hline Total volume $\left(\mathrm{m}^{3}\right) /$ house & $129.3(59.4)$ & $58.7(45.7)$ \\
\hline Total openness area $\left(\mathrm{m}^{2}\right) /$ house & $6.6(5.0)$ & $5.7(4.3)$ \\
\hline Corrected openness area/volume & 0.051 & 0.098 \\
\hline No. of metofluthrin strips $/ \mathrm{m}^{2}$ & 0.31 & 0.52 \\
\hline Amount of active ingredient $(\mathrm{mg}) / \mathrm{m}^{2}$ & 191 & 320 \\
\hline Effective duration (wk) & 8 & $>18$ \\
\hline
\end{tabular}

\footnotetext{
${ }^{1}$ Figures in parentheses are standard deviations.

${ }^{2}$ Kawada et al. (2006).

${ }^{3}$ June 20-August 3, 2006 in Bagamoyo; June 20-September 4, 2005 in My Tho.
} 
complex throughout the year. For example, Shiff et al. (1995) reported that the monthly geometric mean numbers of An. gambiae and An. funestus complexes collected per night in Bagamoyo district in 1992 were 59.48 and 1.72 in May and 29.6 and 3.86 in June, respectively. In our report, the densities of An. funestus complex by both CDC trap and pyrethrum spray sheet collection were also low when compared with those of $A n$. gambiae complex; thus, statistical comparison between intervention and control was not performed.

Mosquito traps are convenient and are the principal means for monitoring the adult mosquito populations. In the present study, the CDC trap collection was used as a substitute for the human-baited collection. However, it was noticed that the reduction in the mosquito density was greater when the collection was performed by using the pyrethrum spray sheet than when the CDC trap was used. This may suggest that the mode of action of metofluthrin via spatial repellency, i.e., metofluthrin producing knockdown or repellency of mosquitoes that entered the houses after a certain time period, is presumed to be less than $20 \mathrm{~min}$, because the $\mathrm{KT}_{50} \mathrm{~S}$ for mosquitoes released in a confined nonventilated 28- $\mathrm{m}^{3}$ room were 10-16 min (Matsuo et al. 2005). Kawada et al. (2004a, 2004b, 2005a, 2005b, 2006) reported that mosquitoes were repelled by airborne metofluthrin vapors by the 2 main modes of pyrethroid action, i.e., knockdown activity and biting inhibition or disruption of orientation toward the host. Of these, the latter may be categorized as a sublethal and "delayed" effect that results from neural excitement, which appears to occur at an earlier stage of pyrethroid toxicity (MacIver 1964, Winney 1975, Birley et al. 1987). It might be impossible for us to distinguish the metofluthrin-affected mosquitoes among those collected by the CDC traps, because the CDC trap "vacuums" all mosquitoes flying near the fan, regardless of them being affected by metofluthrin. Collection by pyrethrum spray sheet, as reported in the present article, and direct collection of resting mosquitoes with aspirator, as reported in previous articles when we evaluated efficacy against Ae. aegypti and $C x$. quinquefasciatus (Kawada et al. 2004a, 2004b, $2005 \mathrm{a}, 2005 \mathrm{~b}, 2006$ ) are probably the better collection methods that result in a more reasonable evaluation of a spatial repellent such as metofluthrin. Recently, the authors performed a comparison of 2 collection methods against $A e$. aegypti in Vietnam, 1 using the BG-Sentinel ${ }^{\mathrm{TM}}$ trap and the other resting-mosquito collection with aspirators. The test results were almost the same as in the present report; that is, the direct collection of resting mosquitoes was the better of the 2 collection methods (Kawada et al. unpublished).
Kawada et al. (2006) reported that an increase in the average room temperature and a decrease in the opening areas of the rooms treated with metofluthrin-impregnated strips exerted an increased spatial repellent effect. The increase in temperature might increase the evaporation rate, and the decrease in the opening area might retain the active ingredient inside the rooms, thereby resulting in an increased concentration of metofluthrin in the air. The corrected opening area/ volume in the houses in Bagamoyo was nearly twice that of the houses in My Tho city (Table 2). A large opening area would potentially facilitate the entry of endophilic and nocturnal mosquitoes, and the presence of large and numerous open eaves in the typical rural African houses are considered to be one of the most important entrances for invasion by An. gambiae during the night. Snow (1987) reported that the invasion by An. gambiae, An. melas Theobald, and Mansonia sp. into the experimental huts was slightly affected by increasing the wall height. Lindsay et al. (2003) reported that the entry of $A n$. gambiae into houses was reduced by $37 \%$ subsequent to the closure of the eaves. Similarly, a significant contribution of open eaves to an increase in mosquito invasion was reported by Pålsson et al. (2004). We therefore argue that the large opening areas of the houses in Bagamoyo might have negatively affected the spatial repellent efficacy of metofluthrin.

This study was able to confirm the long-lasting spatial repellent efficacy of metofluthrin-impregnated plastic strips against the An. gambiae complex. The effective duration of repellency of this new device appears to be extended by increasing the dosage, although the trial sites and the target mosquitoes in the 2 trials were different (Table 2). The effective duration of repellency $(>18 \mathrm{wk})$ is believed to be sufficient for the practical application of these devices, considering the convenient replacement of the formulation. Further improvements related to the manufacturing of plastic strips, such as optimization of the composition and the density of the plastic polymer in order to reduce the loss of the active ingredient, may enable the development of an optimum formulation that would result in a longer effective duration and lower treatment cost.

\section{ACKNOWLEDGMENTS}

We express our deep gratitude to Nobuko Tuno and Mayumi Abe, Institute of Tropical Medicine, Nagasaki University, Nagasaki, Japan, for assistance in this study. Special thanks are extended to the people of the Bagamoyo villages who participated in this evaluation. This study is supported by the joint research fund between 
Nagasaki University and Sumitomo Chemical Co., Ltd., Osaka, Japan.

\section{REFERENCES CITED}

Birley MH, Mutero CM, Turner IF, Chadwick PR. 1987. The effectiveness of mosquito coils containing esbiothrin under laboratory and field conditions. Ann Trop Med Parasitol 81:163-171.

Davis JR, Hall T, Chee EM, Majala A, Minjas JN, Shiff CJ. 1995. Comparison of sampling anopheline mosquitoes by light-trap and human-bait collections indoors at Bagamoyo, Tanzania. Med Vet Entomol 9:249-255.

Kawada H, Maekawa Y, Tsuda Y, Takagi M. 2004a. Laboratory and field evaluation of spatial repellency with metofluthrin-impregnated paper strip against mosquitoes in Lombok Island, Indonesia. J Am Mosq Control Assoc 20:292-298.

Kawada H, Maekawa Y, Tsuda Y, Takagi M. 2004b. Trial of spatial repellency of metofluthrin-impregnated paper strip against Anopheles and Culex in shelters without walls in Lombok, Indonesia. J Am Mosq Control Assoc 20:434-437.

Kawada H, Maekawa Y, Takagi M. 2005a. Field trial of the spatial repellency of metofluthrin-impregnated plastic strip against mosquitoes in shelters without walls (Beruga) in Lombok, Indonesia. J Vector Ecol 30:181-185.

Kawada H, Yen Ng T, Hoa Ng T, Sang Tr M, Dan Ng V, Takagi M. 2005b. Field evaluation of spatial repellency with metofluthrin impregnated resin strips against mosquitoes in Hai Phong city, Vietnam. Am J Trop Med Hyg 73:350-353.

Kawada H, Iwasaki T, Loan LL, Tien TK, Mai NTN, Shono Y, Katayama Y, Takagi M. 2006. Field evaluation of spatial repellency of metofluthrinimpregnated latticework plastic strips against Aedes aegypti (L.) and analysis of environmental factors affecting its efficacy in My Tho city, Tien Giang, Vietnam. Am J Trop Med Hyg 75:1153-1157.

Lindsay SW, Jawara M, Paine K, Pinder M, Walraven GEL, Emerson PM. 2003. Changes in house design reduce exposure to malaria mosquitoes. Trop Med Int Health 8:512-517.

MacIver DR. 1964. Mosquito coils. Part II. Studies on the action of mosquito coil smoke on mosquitoes. Pyrethrum Post 7:7-14.

Matsuo N, Ujihara K, Shono Y, Iwasaki T, Sugano M, Yoshiyama T, Uwagawa S. 2005. Discovery and development of a novel pyrethroid insecticide "Metofluthrin (Sumione ${ }^{\circledR}$, Eminence ${ }^{\circledR}$ ).” Sumitomokagaku II:4-16 (in Japanese).

Pålsson K, Jaenson TGT, Dias F, Laugen AT, Björkman A. 2004. Endophilic Anopheles mosquitoes in Guinea Bissau, West Africa, in relation to human housing conditions. J Med Entomol 41:746-752.

Service MW. 1993. Sampling the adult resting population. In: Service MW, ed. Mosquito ecology field sampling methods. 2nd ed. London: Elsevier Applied Science. p 210-290.

Shiff CJ, Minjas JN, Hall T, Hunt RH, Lyimo S, Davis R. 1995. Malaria infection potential of anopheline mosquitoes sampled by light trapping indoors in coastal Tanzania villages. Med Vet Entomol 9:256-262.

Snow WF. 1987. Studies of house-entering habits of mosquitoes in The Gambia, West Africa: experiments with prefabricated huts with varied wall apertures. Med Vet Entomol 1:9-21.

Temu EA, Minjas JN, Coetzee M, Hunt RH, Shiff CJ. 1998. The role of four anopheline species (Diptera: Culicidae) in malaria transmission in coastal Tanzania. Trans $R$ Soc Trop Med Hyg 92:152-158.

Ujihara K, Mori T, Iwasaki T, Sugano M, Shono Y, Matsuo N. 2004. Metofluthrin: a potent new synthetic pyrethroid with high vapor activity against mosquitoes. Biosci Biotechnol Biochem 68:170-174.

Winney R. 1975. Pyrethrins and pyrethroids in coils-a review. Pyrethrum Post 13:17-22. 